\title{
Validação de metodologia analítica para a determinação de derivados do ácido o- hidroxicinâmico de Echinodorus grandiflorus
}

\author{
LOPES, G.C. ${ }^{1}$; SANTOS, P.V.P. ${ }^{1}$; DICIAULA, M.C. ${ }^{1}$; BLAINSKI, A. ${ }^{1}$; GUTIERRE, M.A.M. ${ }^{2}$; MELLO, J.C.P. ${ }^{1 *}$ \\ ${ }^{1}$ Departamento de Farmácia, ${ }^{2}$ Departamento de Biologia, Universidade Estadual de Maringá, Avwnida Colombo, \\ 5790, CEP: 87020-900, Maringá-Brasil *mello@uem.br
}

\begin{abstract}
RESUMO: Este artigo descreve o desenvolvimento e a validação de método espectrofotométrico UV-Visível para quantificação de derivados do ácido o-hidroxicinâmico em folhas de Echinodorus grandiflorus. O método demonstrou ser linear $\left(r^{2}=0,9974\right)$, preciso (DPR $\left.<15 \%\right)$ na análise de matriz complexa e exata (recuperação $=107,56 \%$ ).
\end{abstract}

Palavras-chave: Echinodorus grandiflorus, espectrofotometria UV-Vis, derivados do ácido ohidroxicinâmico, controle de qualidade, validação de metodologia analítica

\begin{abstract}
Validation of analytical methodology for the determination of derivatives of o-hydroxycinnamic acid from Echinodorus grandiflorus. This paper describes the development and the validation of an UV-Vis spectrophotometric method for the quantification of derivatives of o-hydroxycinnamic acid in leaves of Echinodorus grandiflorus. The method showed to be linear $\left(\mathrm{r}^{2}=\right.$ $0.9974)$, precise $(\mathrm{RSD}<15 \%)$ in the analysis of complex matrix and accurate (recovery $=107.56 \%)$.
\end{abstract}

Key words: Echinodorus grandiflorus, UV-Vis spectrophotometry, o-hydroxycinnamic acid derivatives, quality control, validation of analytical methodology

\section{INTRODUÇÃO}

Entre as inúmeras espécies vegetais de interesse medicinal encontram-se plantas do gênero Echinodorus pertencente à família Alismataceae que ocorrem, predominantemente, em terrenos brejosos e ácidos de todo o continente Americano. Em meio a quase 27 espécies de Echinodorus, as mais amplamente estudadas do ponto de vista químico e biológico são Echinodorus grandiflorus (Cham. \& Schltdl.) Mich. e Echinodorus macrophyllus (Kunth) Mich., ambas conhecidas popularmente no Brasil como chapéu-de-couro (Lorenzi, 2000). Tradicionalmente as folhas dessas espécies têm sido empregadas pela população por propriedades medicinais, tais como diurética, depurativa e tônica (Lorenzi \& Matos, 2002).

A composição química de folhas das espécies de Echinodorus avaliada até o presente sugere um conteúdo químico constituído por ácido trans-aconítico, glicosilflavonas e ácido hidroxitartárico (Schnitzler et al., 2007), sesquiterpenos (Pimenta et al., 2006) e, principalmente, diterpenos dos tipos clerodano, cembrano e labdano (Manns \& Hartmann, 1993; Tanaka et al., 1997; Costa et al., 1999; Kobayashi et al., 2000a; b), substâncias estas presentes nos extratos avaliados e que, provavelmente, estão envolvidas nas atividades biológicas relatadas até o momento, tais como atividade anti-hipertensiva (Lessa et al., 2008), imunossupressora (Pinto et al., 2007), vasodilatadora (Tibiriçá et al., 2007), hipocolesterolemiante (Cardoso et al., 2005), antimicrobiana (Coelho de Souza et al., 2004), diurética (Ribeiro et al., 1988; Cardoso et al., 2003) e analgésica e antiinflamátoria (Cardoso et al., 2003; Dutra et al., 2006). No entanto, Garcia et al. (2010) demonstraram que a fração contendo derivados do ácido tartárico não possui atividade antiedematogênica. Pela importância terapêutica das espécies medicinais do gênero Echinodorus, estudos toxicológicos têm sido realizados (Lopes et al., 2000; Moreti et al., 2006; Da Silva et al., 2010) e sugerem a ausência de toxicidade nos critérios avaliados.

Apesar do grande número de estudos sobre a composição química e a atividade biológica de espécies do gênero Echinodorus, não foram encontrados na literatura dados de controle de qualidade físico-química sobre as espécies usadas para fins medicinais.

Considerando a necessidade do mercado e

Recebido para publicação em 10/07/2010

Aceito para publicação em 01/01/2011

Rev. Bras. PI. Med., Botucatu, v.14, n.3, p.500-505, 2012. 
a falta de especificação de controle de qualidade, o presente trabalho destaca o desenvolvimento e a validação de metodologia analítica para a determinação de derivados do ácido o-hidroxicinâmico de Echinodorus grandiflorus (Cham. \& Schltdl.) Mich.

\section{MATERIAL E MÉTODO}

Material vegetal: Folhas rasuradas de Echinodorus grandiflorus (Cham. \& Schltdl.) Mich. foram fornecidas por Santosflora Comércio de Ervas Ltda (São Paulo, Brasil), com laudo da autenticidade fornecido pela empresa. A espécie vegetal foi identificada pelo Prof. Dr. Lin Chau Ming e a exsicata encontra-se depositada no Herbário da Universidade Estadual de Maringá sob o número HUEM 20810. A autenticidade da droga rasurada foi estabelecida por comparação pela Profa Dra Maria Auxiliadora Milaneze Gutierre e duplicatatas foram depositadas no Herbário da Universidade Estadual de Maringá. Adroga vegetal foi moída em moinho de martelos (Tigre ASN5) e a granulometria definida.

Instrumentação: Medições espectrofotométricas foram realizadas em um espectrofotômetro Shimadzu UV-Vis, modelo PC-1650, com cubetas de quartzo QS.

Preparação da amostra: Pesaram-se cerca de $0,500 \mathrm{~g}$ da droga vegetal pulverizada $(0,210$ $\mathrm{mm})(\mathrm{n}=3)$, adicionaram-se $90 \mathrm{~mL}$ de álcool $50 \%(\mathrm{v} /$ v) em balão de fundo redondo de $250 \mathrm{~mL}$ que foi submetido a refluxo por $30 \mathrm{~min}$. Após esfriar, os extratos foram filtrados para balão volumétrico de 100 $\mathrm{mL}$, lavando-se o balão de fundo redondo e o filtro com $10 \mathrm{~mL}$ de álcool $50 \%$ para o balão volumétrico. Por fim, o volume da solução foi ajustado com álcool $50 \%$ para o balão volumétrico (SM).

Determinação de derivados do ácido ohidroxicinâmico: Adicionaram-se volumetricamente, para balão volumétrico de $10 \mathrm{~mL}, 1 \mathrm{~mL}$ da SM, $2 \mathrm{~mL}$ de ácido clorídrico $0,5 \mathrm{~mol} \mathrm{~L}^{-1}, 2 \mathrm{~mL}$ da solução reagente ( $10 \mathrm{~g}$ de nitrito de sódio $R, 10 \mathrm{~g}$ de molibdato de sódio $R$ em $100 \mathrm{~mL}$ de água destilada) e $2 \mathrm{~mL}$ de solução de hidróxido de sódio $\mathrm{R} 8 \%$, ajustando o volume com álcool $50 \%$ para o balão volumétrico. A absorbância foi medida imediatamente após o preparo da solução no comprimento de onda de $525 \mathrm{~nm}$, utilizando como solução de compensação, $1 \mathrm{~mL}$ da $\mathrm{SM}, 2 \mathrm{~mL}$ de ácido clorídrico $0,5 \mathrm{~mol} \mathrm{~L}^{-1}$ e $2 \mathrm{~mL}$ de solução de hidróxido de sódio $\mathrm{R} 8 \%$ em balão volumétrico de $10 \mathrm{~mL}$ que foi completado com álcool $50 \%$ (European Pharmacopoeia, 2005). A análise foi realizada em triplicata.

A porcentagem do teor de derivados do ácido o-hidroxicinâmico expresso em verbascosídeo (absortividade específica=185) foi calculada pela expressão:

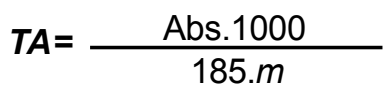

Em que:

$T A=$ Teor de derivados do ácido 0 hidroxicinâmico (\%)

Abs = Absorbância da solução amostra;

$m=$ massa da amostra utilizada no ensaio, em gramas, considerando a determinação de água.

Parâmetros de validação: Para a validação do método foram seguidas as determinações da Agência Nacional de Vigilância Sanitária (Brasil, 2003), com a construção da curva de analítica da amostra e a obtenção dos parâmetros analíticos: exatidão, precisão e reprodutibilidade interlaboratorial.

Linearidade: $O$ teste de linearidade foi realizado através da construção de curva analítica da amostra. Foram empregadas cinco diferentes concentrações de amostra, 0,25; 0,40; 0,50; 0,75; $0,90 \mu \mathrm{g} \mathrm{mL}^{-1}$. Os valores de absorbância medidos foram tratados por métodos estatísticos para a determinação da linearidade do método através do coeficiente de variação relativo e da análise de regressão linear. Todos os procedimentos foram realizados em triplicata.

Precisão: Para o estudo de repetibilidade (precisão intra-corrida) foi utilizada a concentração de $0,50 \mu \mathrm{g} \mathrm{mL}^{-1}$ (concentração teste) em seis determinações, realizadas pelo mesmo analista, no mesmo laboratório, com a finalidade de verificar se os resultados encontravam-se dentro da diferença máxima aceitável. A precisão intermediária foi expressa através de variações do mesmo laboratório (equipamento), em dias diferentes e determinadas em três replicatas.

Exatidão: A exatidão do método foi determinada pela concentração do ativo na amostra em três diferentes níveis $\left(0,75 ; 0,82\right.$ e $\left.0,90 \mu \mathrm{g} \mathrm{mL}^{-1}\right)$. Foram realizadas três determinações de cada nível. Para a concentração teórica foi utilizada a média das análises de precisão da amostra na concentração de $0,50 \mu \mathrm{g} \mathrm{mL}^{-1}$ (concentração teste), uma vez que se trata de um conjunto de substâncias (derivados do ácido o-hidroxicinâmico) e o resultado da exatidão foi dado pela expressão:

Exatidão $=\frac{(\text { concentração experimental })}{\text { (concentração téorica })} \times 100$ 
Ensaio interlaboratorial: Nesta avaliação participaram três laboratórios, sendo eles dos estados do Paraná, Santa Catarina e Rio Grande do Sul (Brasil): Departamento de Farmácia e Farmacologia, Universidade Estadual de Maringá, Paraná (UEM); Centro de Ciências da Saúde, Universidade do Vale do Itajaí, Santa Catarina (UNIVALI); Departamento de Farmácia Industrial, Universidade Federal de Santa Maria, Rio Grande do Sul (FATECIENS). Os estudos foram organizados e os protocolos foram criados de acordo com a norma ISO 5725 para determinar a repetibilidade e reprodutibilidade do método de medição.

As amostras foram especialmente preparadas para este fim no Laboratório de Farmacognosia da Universidade Estadual de Maringá, sendo distribuídas com a homogeneidade comprovada e material com granulometria de $0,210 \mathrm{~mm}$. Cada laboratório recebeu uma amostra de $10 \mathrm{~g}$ e efetuou três replicatas.

Analise estatística: A análise estatística dos dados foi realizada através de análise de variância ANOVA, onde os resultados são considerados significativos quando a probabilidade é inferior a $5 \%$ $(p<0,05)$ e pelo teste $t$ de Student, com nível de significância $\alpha=0,05$. A avaliação estatística dos resultados foi realizada através do Software Prisma ${ }^{\circledR}$, STATISTICA ${ }^{\circledR}$ e Excel ${ }^{\circledR}$.

\section{RESULTADO E DISCUSSÃO}

Diversas técnicas podem ser empregadas para a detecção e doseamento de substâncias químicas em amostras vegetais, tais como a cromatografia líquida de alta eficiência (CLAE), cromatografia gasosa (CG), cromatografia líquida associada à espectrometria de massas (CL-EM), espectrofotometria UV/Vis, entre outras.

A espectrofotometria é um processo de medida que basicamente emprega as propriedades dos átomos e moléculas de absorverem e/ou emitirem energia eletromagnética em uma das regiões do espectro eletromagnético, sendo uma das técnicas analíticas mais amplamente empregadas, em função da robustez, custo relativamente baixo, e grande número de aplicações desenvolvidas (Lobinski \& Marczenko, 1992).

O desenvolvimento de metodologias menos onerosas, mais facilmente exequível e que normalmente empregam menor aparato instrumental, requerem a realização de estudos de validação destas técnicas para cada espécie vegetal específica a fim de garantir a confiabilidade nos resultados obtidos (Pelozo et al., 2008; Silva-Corazza et al., 2010). As metodologias de ordem geral não têm apresentado resultados coerentes quando empregadas a diferentes famílias, gêneros e até espécies de plantas, que apresentam na composição os mesmos grupos de substâncias.

A faixa de trabalho do método em estudo foi determinada através do intervalo de concentrações das amostras usadas na composição da curva analítica e que, obrigatoriamente, apresentou linearidade.

No parâmetro de linearidade do extrato, os pontos da curva foram plotados em um gráfico de concentração ( $\mu \mathrm{g} \mathrm{mL}^{-1}$ ) versus absorbância e obtida a curva analítica (Tabela 1) e a respectiva equação da reta pela regressão linear usando o método dos mínimos quadrados, bem como o correspondente coeficiente de correlação linear (Figura 1).

A construção da curva analítica através do método dos mínimos quadrados permite a quantificação do analito caso a curva obedeça a todos os critérios de desempenho. Portanto, a avaliação da homogeneidade na variância dos resíduos (homo/ heterocedasticidade) indica se em todo o intervalo de trabalho considerado o método dos mínimos quadrados pode ou não ser usado na análise de regressão da reta. De acordo com a planilha de cálculo desenvolvida para avaliação dos resultados analíticos e tratamento estatístico da linearidade do método, não houve falta de ajuste no intervalo estudado, demonstrando a linearidade do método. Ou seja, os resultados demonstraram que o método foi linear, sugerindo a eficiência no processo de extração empregado e na concentração do reagente colorimétrico utilizado na avaliação dos derivados do ácido o-hidroxicinâmico.

TABELA 1. Resultado do estudo de linearidade da amostra de E. grandiflorus.

\begin{tabular}{ccc}
\hline $\begin{array}{c}\text { Concentração } \\
\mu \mathbf{g ~ m L}^{-1}\end{array}$ & $\begin{array}{c}\text { Valor médio de absorbância } \\
(\mathbf{n}=3)[\bar{x} \pm d p(\mathbf{C V} \%)]\end{array}$ & $\begin{array}{c}\text { TA }(\%) \\
{[\bar{x} \pm d p(\mathbf{C V} \%)]}\end{array}$ \\
\hline 0,25 & $0,152 \pm 0,003(2,01)$ & \\
0,40 & $0,244 \pm 0,012(4,79)$ & \\
0,50 & $0,314 \pm 0,008(2,76)$ & $3,40 \pm 0,141(4,14)$ \\
0,75 & $0,483 \pm 0,011(2,39)$ & \\
0,90 & $0,595 \pm 0,004(0,61)$ & \\
\hline
\end{tabular}

Rev. Bras. PI. Med., Botucatu, v.14, n.3, p.500-505, 2012. 


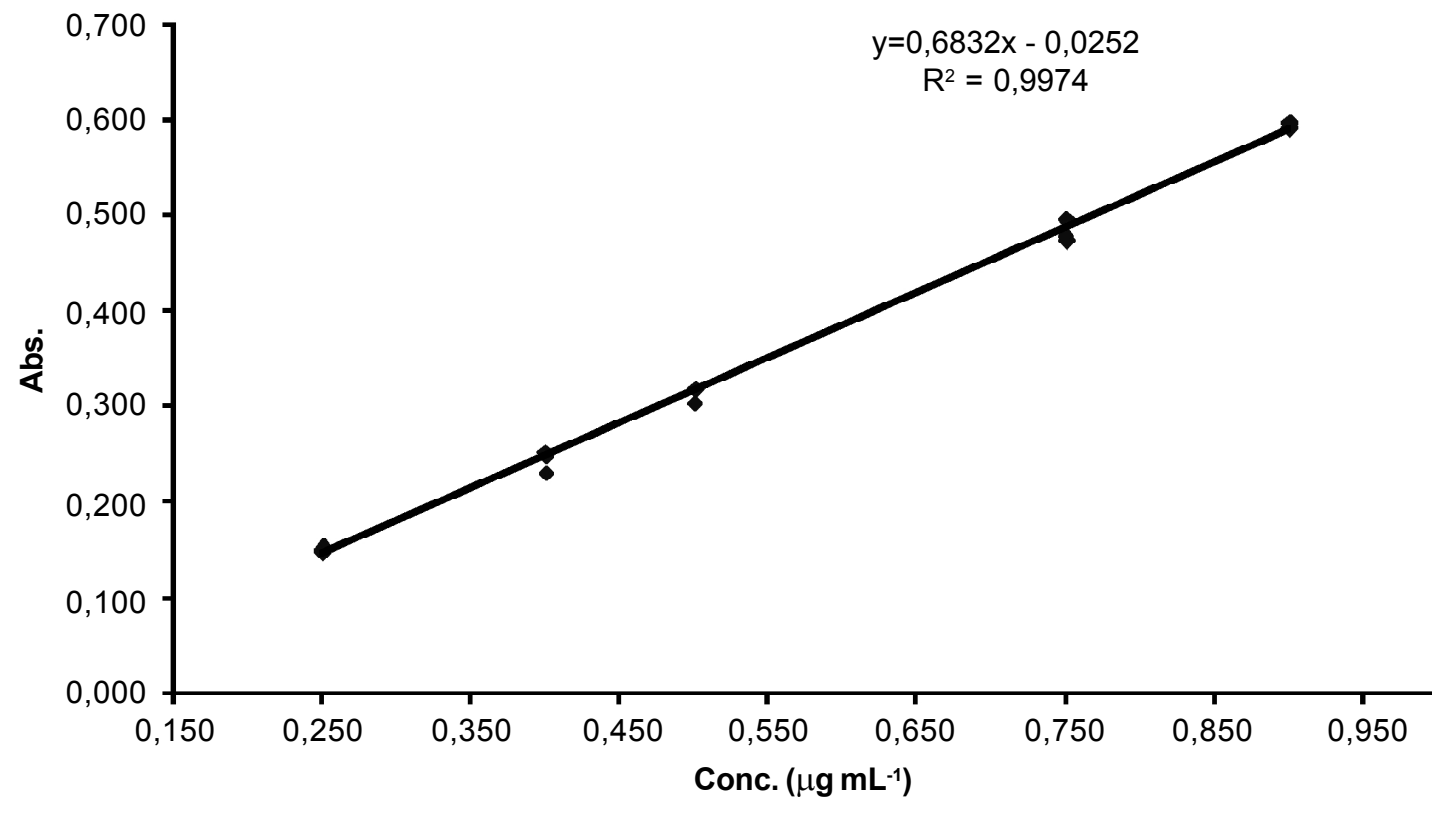

FIGURA 1. Curva analítica de Echinodorus grandiflorus. Valor mínimo do coeficiente de correlação ("r2") estipulado pela RE no 899: 0,9900.

O resultado do teste de repetibilidade está representado na Tabela 2. A precisão intermediária, que avalia a variação de ensaios realizados em dias diferentes, mostrou valor de coeficiente de variação de $15 \%$. Através do teste t de Student foi avaliada a possível existência de diferença estatisticamente significativa entre as médias obtidas para os ensaios realizados em dias diferentes. No entanto, o t calculado $(-0,9363)$ foi menor do que o $t$ tabelado $(2,776)$, demonstrando não existir diferença estatisticamente significativa entre as médias, ou seja, os resultados obtidos expressam o mesmo valor no diferentes dias $(p<0,05)$.

TABELA 2. Ensaio de repetibilidade para determinação de derivados do ácido ohidroxicinâmico, expressos como verbascosídeo em E. grandiflorus.

\begin{tabular}{lccc}
\hline Amostra & Abs. & TA $(\%)$ & {$[\bar{x} \pm d p(\mathrm{CV} \%)]$} \\
\hline EG1 & 0,319 & 3,73 & \\
EG2 & 0,267 & 3,12 & $3,46 \pm 0,25(7,4)$ \\
EG3 & 0,304 & 3,55 & \\
\hline
\end{tabular}

Valor máximo de CV\% estipulado pela RE 899: 15\%

A exatidão foi verificada para três níveis de amostragem, baixa, média e alta. Os dados experimentais obtidos revelaram recuperação média de 107,56 $\pm 4,85(4,51)$ e que o maior coeficiente de variação foi de $1,95 \%$ (Tabela 3 ). Os resultados do estudo de exatidão demonstram que possíveis variações da concentração de derivados do ácido ohidroxicinâmico, em virtude de variações no padrão da matéria-prima, podem ser prontamente quantificadas pelo método, portanto, o método analítico desenvolvido é suficientemente exato.

A informação interlaboratorial é extremamente útil para estimar a incerteza da medição visto que reflete a variação do desempenho do método analítico em função de fatores extremamente importantes, alguns dos quais dificilmente estudados em ambiente intralaboratorial. Além disso, o benefício mais importante a ser usufruído pelos participantes é ter meios de se comparar com laboratórios similares e identificar problemas entre os mesmos quando, trabalhando isolados, não poderiam descobrir (Chui et al., 2009).

No protocolo criado para a análise interlaboratorial foi solicitado a cada laboratório que fizesse três leituras da amostra enviada. Além disso, regulamentou-se que os laboratórios participantes especificassem as dificuldades encontradas na execução do método e no procedimento de análise.

O resultado obtido para o ensaio interlaboratorial está descrito na Tabela 4 . O método é reprodutível, uma vez que, através do ensaio interlaboratorial entre os participantes obteve-se desvio padrão relativo de $9,64 \%$, sendo este valor inferior ao preconizado como limite máximo de significância segundo o Guia para Validação de Métodos Analíticos e Bioanalíticos (RE 899/2003) (Brasil, 2003) para drogas com matriz complexa. Comparando-se os valores entre as Instituições UEM e FATECIENS o desvio padrão relativo foi de $3,00 \% \mathrm{e}$ entre a UEM e UNIVALI o DPR foi de $10,31 \%$. 
TABELA 3. Resultados da exatidão para três níveis de amostragem na determinação de derivados do ácido ohidroxicinâmico em E. grandiflorus.

\begin{tabular}{|c|c|c|c|c|}
\hline $\begin{array}{c}\text { Amostragem } \\
\left(\mu \mathrm{g} \mathrm{mL}^{-1}\right)\end{array}$ & \multicolumn{3}{|c|}{ Recuperação (\%) } & {$[\bar{x} \pm d p(\mathrm{CV} \%)]$} \\
\hline $\begin{array}{l}0,75 \\
\text { (baixa) }\end{array}$ & 104,04 & 101,51 & 100,25 & $101,93 \pm 1,57(1,54)$ \\
\hline $\begin{array}{l}0,82 \\
\text { (média) }\end{array}$ & 106,39 & 111,36 & 107,61 & $108,45 \pm 2,11(1,95)$ \\
\hline $\begin{array}{l}0,90 \\
\text { (alta) }\end{array}$ & 111,47 & 111,85 & 113,57 & $112,29 \pm 0,91(0,81)$ \\
\hline & & & Total & $107,56 \pm 4,85(4,51)$ \\
\hline
\end{tabular}

TABELA 4. Resultado do ensaio interlaboratorial para a determinação do teor de derivados do ácido ohidroxicinâmico em E. grandiflorus.

\begin{tabular}{lc}
\hline Laboratório & TA $(\%)[\bar{x} \pm d p(\mathbf{C V} \%)]$ \\
\hline UEM & $3,46 \pm 0,25(7,36)$ \\
UNIVALI & $2,99 \pm 0,003(0,11)$ \\
FATECIENS & $3,61 \pm 0,16(4,53)$ \\
\hline
\end{tabular}

O método desenvolvido cumpriu todos os parâmetros de validação e mostrou-se, rápido, viável, e de baixo custo. Assim, ele pode ser aplicado por farmácias de manipulação e laboratórios de pequeno porte para a rotina de análise de controle de qualidade de Echinodorus grandiflorus.

\section{AGRADECIMENTO}

Os autores agradecem a ANVISA (Agência Nacional de Vigilância Sanitária) e a Farmacopeia Brasileira. O estudo foi financiado pelo edital no 001/ 2007-ANVISA, Farmacopeia Brasileira. Agradecemos também a Profa Dra Melânia Palermo Manfron e Luciana Cátia Block pela colaboração no ensaio interlaboratorial.

\section{REFERÊNCIA}

BRASIL, Ministério da Saúde, Agência Nacional de Vigilância Sanitária, Resolução Específica (RE) 899 de 29 de maio de 2003. Determina a publicação do Guia para validação de métodos analíticos e bioanalíticos, Diário Oficial da União, 2003.

CARDOSO, G.L.C. et al. Avaliação das atividades antinociceptiva, antiinflamatória e diurética de chapéude-couro (Echinodorus grandiflorus [Cham. e Schl] Mitch.,
Alismataceae). Revista Brasileira de Farmácia, v.84, n.1, p.5-7, 2003.

CARDOSO, G.L.C. et al. Avaliação da atividade do chapéu-de-couro (Echinodorus grandiflorus [Cham. \& Schl.] Mitch., Alismataceae) sobre os níveis plasmáticos de colesterol em camundongos. Revista Brasileira de Farmácia, v.86, n.3, p.95-6, 2005.

CHUI, Q.S.H. et al. Parâmetros r e R obtidos de programa interlaboratorial - como usá-los. Química Nova, v.32, n.8, p.2209-13, 2009.

COELHO DE SOUZA, G. et al. Ethnopharmacological studies of antimicrobial remedies in the south of Brazil. Journal of Ethnopharmacology, v.90, p.135-43, 2004. COSTA, M. et al. Isolation and synthesis of a new clerodane from Echinodorus grandiflorus. Phytochemistry, v.50, p.117-22, 1999.

DA SILVA, C.J. et al. Evaluation of the genotoxic and cytotoxic effects of crude extracts of Cordia ecalyculata and Echinodorus grandiflorus. Journal of Ethnopharmacology, v.27, n.2, p.445-50, 2010.

DUTRA, R.C. et al. Investigação das atividades analgésica e antiinflamatória do extrato metanólico dos rizomas de Echinodorus grandiflorus. Brazilian Journal of Pharmacognosy, v.16, n.4, p.469-74, 2006.

EUROPEAN PHARMACOPOEIA, 5.ed. Strasbourg: France, European Directorate for the Qualit of Medicines, 2005.

GARCIA, E.F. et al. Antiedematogenic activity and phytochemical composition of preparations from Echinodorus grandiflorus leaves. Phytomedicine, v.18, p.80-6, 2010.

KOBAYASHI, J. et al. Chapecoderins A-C, new labdanederived diterpenoids from Echinodorus macrophyllus. Journal of Natural Products, v.63, p.375-7, 2000a.

KOBAYASHI, J. et al. Echinophyllins A and B, novel nitrogencontaining clerodane diterpenoids from Echinodorus macrophyllus. Tetrahedron Letters, v.41, p.2939-43, 2000b. LESSA, M.A. et al. Antihypertensive effects of crude extracts from leaves of Echinodorus grandiflorus. Fundamental \& Clinical Pharmacology, v.22, p.161-8, 2008.

LOBINSKI, R.; MARCZENKO, Z. Recent advances in ultraviolet-visible spectrophotometry. Critical Reviews in Analytical Chemistry, v.23, n.1-2, p.55-111, 1992.

LOPES, L.C. et al. Toxicological evaluation by in vitro and 
in vivo assays of an aqueous extract prepared from Echinodorus macrophyllus leaves. Toxicology Letters, v.116, p.189-98, 2000.

LORENZI, H. Plantas daninhas do Brasil: terrestres, aquáticas, parasitas e tóxicas. 4.ed. Nova Odessa: Instituto Plantarum de Estudos da Flora, 2000. 672p.

LORENZI, H.; MATOS, F.J.A. Plantas medicinais no Brasil: nativas e exóticas. Nova Odessa: Instituto Plantarum de Estudos da Flora, 2002. 512p.

MANNS, D.; HARTMANN, R. Echinodol: a new cembrene derivative from Echinodorus grandiflorus. Planta Medica, v.59, p.465-6, 1993.

MORETI, D.L.C. et al. Estudo hematológico em ratos sob ação de plantas medicinais. XXXVII. Echinodorus macrophyllus (Kunth.) Mitch. Revista Brasileira de Plantas Medicinais, v.8, n.4, p.162-4, 2006.

PELOZO, M.I.G. et al. Spectrophotometric determination of tannins and caffeine in preparations from Paullinia cupana var. sorbilis. Brazilian Archives of Biology and Technology, v.51, n.3.p.447-51, 2008.

PIMENTA, D.S. et al. Essential oil from two populations of Echinodorus grandiflorus (Cham. \& Schltdl.) Micheli (Chapéu de couro). Anais da Academia Brasileira de
Ciências, v.78, n.4, p.623-8, 2006.

PINTO, A.C. et al. Immunosuppressive effects of Echinodorus macrophyllus aqueous extract. Journal of Ethnopharmacology, v.111, p.435-9, 2007.

RIBEIRO, R.A. et al. Acute diuretic effects in conscious rats produced by some medicinal plants used in the state of São Paulo, Brasil. Journal of Ethnopharmacology, v.24, n.1, p.19-29, 1988.

SCHNITZLER, M. et al. Trans-aconitic acid, glucosylflavones and hydroxycinnamoyltartaric acids from the leaves of Echinodorus grandiflorus ssp. aureus, a Brazilian medicinal plant. Brazilian Journal of Pharmacognosy, v.17, n.2, p.149-54, 2007.

SILVA-CORAZZA, P.E.R. et al. Pharmaceutical topical gel: development and validation of a UV spectrophotometric method for determination of polyphenols. Latin American Journal of Pharmacy, v.29, n.5, p.1-5, 2010.

TANAKA, C.M.A. et al. A membrane from Echinodorus grandiflorus. Phytochemistry, v.44, n.8, p.1547-9, 1997. TIBIRIÇÁ, E. et al. Pharmacological mechanisms involved in the vasodilator effects of extracts from Echinodorus grandiflorus. Journal of Ethnopharmacology, v.111, p.50-5, 2007. 